\title{
On two invariants of divisorial valuations at infinity
}

\author{
Alexander Borisov
}

Received: 13 February 2013 / Accepted: 12 July 2013 / Published online: 10 August 2013

(C) Springer Science+Business Media New York 2013

\begin{abstract}
The two-dimensional case of the famous Jacobian conjecture of O.-H. Keller asserts that every unramified polynomial self-map of an affine plane is invertible. Many geometric approaches to this conjecture involve divisorial valuations of the field $\mathbb{C}(x, y)$, centered outside of the affine plane. Two integer invariants of these valuations naturally appear in this context. In this paper we study these invariants using combinatorics of weighted graphs. In particular, we prove that whenever both invariants are fixed, the corresponding valuations form a finite number of families up to plane automorphisms.
\end{abstract}

Keywords Divisorial valuation · Weighted graph · Blowup

\section{Introduction}

The main motivation for this paper comes from one of the oldest unsolved problems in algebraic geometry, the Jacobian conjecture.

Jacobian conjecture (O.-H. Keller, 1939) Suppose $n$ is a natural number, and $f_{1}, \ldots, f_{n}$ are complex polynomials in $x_{1}, \ldots, x_{n}$, such that the Jacobian, $\operatorname{det}\left(\partial f_{i} / \partial x_{j}\right)$, is a non-zero constant. Then the map

$$
\left(x_{1}, \ldots, x_{n}\right) \mapsto\left(f_{1}\left(x_{1}, \ldots, x_{n}\right), \ldots, f_{n}\left(x_{1}, \ldots, x_{n}\right)\right)
$$

is invertible.

In memory of V.A. Iskovskikh.

A. Borisov ( $\bigotimes)$

Department of Mathematics, University of Pittsburgh, 301 Thackeray Hall, Pittsburgh, PA 15260,

USA

e-mail: borisov@pitt.edu 
This is obvious in dimension one (i.e. for $n=1$ ), but is still open for higher $n$, even for $n=2$. Many approaches have been proposed, both for the general conjecture and for its two-dimensional case. The two-dimensional case has been especially well studied, but is still wide open, in sharp contrast to the remarkable progress of modern algebraic geometry, and in particular birational geometry.

One natural approach to the two-dimensional Jacobian conjecture is the following. Suppose a counterexample exists. It gives a rational map from $X=\mathbb{P}^{2}$ to $Y=\mathbb{P}^{2}$. After a sequence of blowups of points outside of the affine plane $\mathbb{A}^{2}$, we can get a surface $Z$ with two morphisms: $\pi: Z \rightarrow X$ (projection onto the origin $P^{2}$ ) and $\phi: Z \rightarrow Y$ (the lift of the original rational map).

Note that $Z$ contains a Zariski open subset isomorphic to $\mathbb{A}^{2}$, and its complement, $\pi^{*}((\infty))$, is a tree of smooth rational curves. The structure of this tree is easy to understand inductively, as it is built from a single curve $(\infty)$ on $\mathbb{P}^{2}$ by a sequence of two operations: blowing up a point on one of the curves or blowing up a point of intersection of two curves. Note that each of these curves defines a valuation on $\mathbb{C}(x, y)$, centered outside of the affine plane. We call these valuations the divisorial valuations at infinity. The map on the set of valuations, induced by $\phi$, sends these valuations at infinity to some valuations on the target field $\mathbb{C}(x, y)$. Many geometric approaches to the two-dimensional Jacobian conjecture use these valuations, either explicitly or via the language of curves (c.f., e.g. [1]).

In a sense, all discrete invariants of divisorial valuations at infinity come from the above described graphs. Two such invariants naturally appear in the context of the Jacobian conjecture.

Definition 1.1 Suppose $Z$ is a surface, obtained from $X=\mathbb{P}^{2}$ by a sequence of blowups outside of $\mathbb{A}^{2}$, and $\pi: Z \rightarrow X$ is the corresponding birational morphism. Suppose $Z \backslash \mathbb{A}^{2}=\bigcup_{i} E_{i}$. Then the $\bar{K}$ label of the divisorial valuation corresponding to $E_{i}$ is the coefficient $a_{i}$ in the formula

$$
\bar{K}_{Z}=\sum_{i} a_{i} E_{i}
$$

where the equality is in the Picard group of $Z$ and $\bar{K}_{Z}=K_{Z}+\sum_{i} E_{i}$ is the logcanonical divisor of the surface $Z$ with the boundary $\sum E_{i}$. Note that the curves $E_{i}$ form a basis of the Picard group of $Z$ and that $a_{i}$ is not affected by any extra blowups, thus is an invariant of the divisorial valuation itself, and not of $E_{i} \subset Z$.

Definition 1.2 Suppose $E_{i}$ and $Z$ are as above. Then the determinant label of the divisorial valuation corresponding to $E_{i}$ is the determinant of the Gram matrix of minus-intersection form on all curves at infinity of $Z$, except $E_{i}$. That is,

$$
d_{E_{i}}=\operatorname{det}\left(-E_{j} \cdot E_{k}\right)_{j, k \neq i} .
$$

We will prove that the determinant label is indeed an invariant of the divisorial valuation, i.e. it does not depend on the choice of $Z$. In some sense, these two invariants are complementary to each other: the first one describes what is going on at $E_{i}$, while 
the second one describes what is going on outside of it. (This was noticed by Bogdan Ion.) We call these invariants "labels", because we label the intersection graphs of curves at infinity on $Z$ with them. The $\bar{K}$ labels behave very well with respect to the blowups, but for the determinant labels the theory is more complicated: to keep track of the determinant labels of vertices under the blowups, one needs to also keep track of the determinant labels of edges of the intersection graph (i.e. the determinants of the matrix associated to the graph with that edge removed). Please see Sect. 3 for the details.

The relevance of the $\bar{K}$ labels to study potential counterexamples to the Jacobian conjecture comes from the following simple observation (cf. [2] for details). If the image of a divisorial valuation at infinity is again a divisorial valuation at infinity (i.e. $\left.\phi\left(E_{i}\right) \cup \mathbb{A}^{2}=\emptyset\right)$, then the $\bar{K}$ label of the valuation of $E_{i}$ is the product of the $\bar{K}$ label of its image and the ramification index of $\phi$ at $E_{i}$. Also, the curves at infinity that are mapped to curves on $\mathbb{A}^{2}$ must have positive $\bar{K}$ labels, equal to the ramification index of $\phi$ there. These conditions provide significant restrictions on the structure of possible counterexamples to the Jacobian conjecture.

The determinant labels also naturally appear as restrictions of the possible counterexamples. In particular, the determinant labels of the curves that are mapped to $\mathbb{A}^{2}$ must be negative. Indeed, if any one of them is positive, the intersection form on the span of the union of all other curves at infinity on $Z$ must be negative definite, which would contradict the fact that $\phi^{*}(\infty)$ has positive self-intersection. For a similar reason, every valuation at infinity that is mapped to a valuation at infinity with negative determinant label must itself have a negative determinant label. Less obviously, one can show that any valuation of a curve mapped to the line at infinity on $Y$ must have a negative determinant label (cf. [2] or [3]).

Note that both the $\bar{K}$ label and the determinant label are invariant under the polynomial automorphisms of $\mathbb{A}^{2}$, where the automorphisms act on valuations in the natural way.

Our main result is the following theorem.

Theorem 1.1 For any pair of integers $(a, b)$, the divisorial valuations at infinity with $\bar{K}$ label $a$ and determinant label $b$ form a bounded family up to the automorphisms of $\mathbb{A}^{2}$. By this we mean that there is a finite number of weighted graphs $\Gamma_{k}$, such that every such valuation is sent by some polynomial automorphism to a valuation given by a curve on a surface $Z$ with one of the graphs $\Gamma_{k}$. Equivalently, there exists some constant $N$ depending on $a$ and $b$ only, such that the curve of every such valuation can be extracted from some $\mathbb{P}^{2}$ compactification of $\mathbb{A}^{2}$ by no more than $N$ blowups.

We also prove the following interesting concrete result.

Theorem 1.2 Every divisorial valuation at infinity with $\bar{K}$ label -2 and determinant label 1 is, up to an automorphism of $\mathbb{A}^{2}$, the valuation of the line at infinity on $\mathbb{P}^{2}$. 
Finally, we prove the following theorem, also motivated by the author's work on the Jacobian conjecture (cf. [2, 3]).

Theorem 1.3 Suppose $X$ and $Z$ are as above, and the divisorial valuations $E_{i}$ and $E_{j}$ have negative $\bar{K}$ label and negative "common determinant" (the determinant of the graph of minus-intersection form on the curves at infinity excluding $E_{i}$ and $E_{j}$ ). Then $E_{i}$ and $E_{j}$ must lie in the same irreducible component of the graph obtained by removing the curve $\pi_{*}^{-1}(\infty)$, the strict pullback of the line at infinity on $\mathbb{P}^{2}$.

While our motivation comes from algebraic geometry, our methods are almost exclusively combinatorial. It would be nice to get a more conceptual, and simpler, proofs of our results. Divisorial valuations at infinity are classical objects, whose study goes back at least to Zariski (cf. [11]). As an excellent example of an algebraic approach to these valuations we should mention the works of Mondal [9].

Besides having applications to the Jacobian conjecture, the valuations are likely to have applications to the dynamics of polynomial maps. Here we should mention the results of Favre and Jonsson [6-8] on more general valuations and their applications. In fact, our invariants are directly related to the main invariants of Favre and Jonsson: thinness and skewness.

By themselves, $\bar{K}$ labels cannot be defined for non-divisorial valuations. However, for any fixed compactification $\mathbb{P}^{2}$ of $\mathbb{A}^{2}$, the ratio of the $\bar{K}$ label and the value of the valuation at the equation of the line at infinity can be extended to a continuous function on the (relative) tree of (normalized) valuations. This is exactly the thinness of Favre and Johsson (cf. [7], Appendix A). Similarly, if the determinant label is normalized by dividing by the square of the value of the valuation at the equation of the line at infinity, it can be extended to the entire relative tree of normalized valuations. In fact, we get precisely the inverse of the skewness invariant of Favre and Jonsson (cf. [6], Chap. 3).

The paper is organized as follows. A short Sect. 2 is devoted to the $\bar{K}$ label. We also define there the notions of parents and ancestors of the given curves or divisorial valuations. Most proofs are only sketched for two reasons: they are easy and they can be found in [2] or [3]. In Sect. 3 we develop the general theory of the determinants of weighted trees and forests. In Sect. 4 we prove Theorems 1.1 and 1.2. In Sect. 5 we prove Theorem 1.3.

\section{The $\bar{K}$ labeling and some definitions}

Suppose $X=P^{2}, Z$, and a birational map $\pi: Z \rightarrow X$ are as in the Introduction. As noted above, the structure of $Z$ is to a large extent determined by the graph of intersections of $E_{i}$. The vertices of this graph correspond to $E_{i}$-s and are usually labeled by $E_{i}^{2}$. The edges correspond to the points of intersections of two different $E_{i}$-s. This graph is a tree. One of the curves $E_{i}$ is $\pi_{*}^{-1}(\infty)$, while all others are mapped to points by $\pi$. The classes of $E_{i}$ form a basis of the Picard group of $Z$.

This graph is not so easy to deal with because blowing up a point changes the selfintersections of the curves passing through it. Inspired by the Minimal Model Program, we consider a different labeling of this graph. We take the augmented canonical 
class of $Z, \bar{K}_{Z}=K_{Z}+\sum_{i} E_{i}$. It can be uniquely written in the Picard group of $Z$ as a linear combination of $E_{i}, \bar{K}_{Z}=\sum_{i} a_{i} E_{i}$. We label the vertices of the intersection graph by these numbers $a_{i}$.

When a new curve at infinity is created by blowing up a point, it does not change the labels of the previously constructed curves. Therefore this label is an invariant of the corresponding divisorial valuation. One can also easily calculate the $\bar{K}$ label of the new curve based on the following Proposition.

Proposition 2.1 When a point is blown up, one of the following two operations is performed to the graph of the exceptional curves:

(1) A new vertex is added to the graph, connected to one of the vertices. It is labeled $a_{i}+1$, where $a_{i}$ is the label of the vertex it is connected to.

(2) A new vertex is introduced on the edge connecting two vertices, breaking up the edge into two edges. The new vertex gets labeled with $a_{i}+a_{j}$, where $a_{i}$ and $a_{j}$ are the labels of the endpoints of the destroyed edge.

Proof The first case corresponds to blowing up a point on one of the curves. The second case corresponds to blowing up an intersection of two curves. The augmented canonical class calculations are straightforward.

Note that the $\bar{K}$ label of the divisorial valuation that corresponds to the line at infinity on $\mathbb{P}^{2}$ is -2 . The following observations are easy to prove by induction. See [2] or [3] for the details.

\section{Proposition 2.2}

(1) For any two adjacent vertices $E_{i}, E_{j}$ of the graph of $Z, \operatorname{gcd}\left(a_{i}, a_{j}\right)=1$. In particular, no two adjacent vertices have even labels.

(2) The subgraph of vertices with negative labels is connected. It is separated from the "positive" vertices by the "zero" vertices. Moreover, the "zero" vertices are only connected to vertices with labels $(-1)$ or 1 .

If $a_{i}$ is nonzero, then one can recover $\left(-E_{i}^{2}\right)$ from $a_{i}$ and the $\bar{K}$ labels of the curves adjacent to $E_{i}$, using the adjunction formula for $E_{i}$. However, when $a_{i}=$ 0 , this is not possible. See [2] or [3] for the details. Note also that the adjunction formula $E_{i}\left(E_{i}+K\right)=-2$ can be rewritten as $E_{i} \bar{K}=(\operatorname{val}(E)-2)$, where $\operatorname{val}(E)$, the valency of $E$, is the number of vertices adjacent to $E$ (i.e. the number of other curves at infinity that intersect $E$ ). Using the adjunction formula as a definition, one can formally define the $\bar{K}$ labels for any weighted graph, if the matrix of that graph (see next section) is invertible. Moreover, when the determinant of this matrix is \pm 1 , these labels will be integers. In general, they will be rational numbers.

We now introduce some definitions that will be freely used later in the paper. These notions are totally classical and go back at least to Zariski; we claim no originality except for the terminology. 
Definition 2.1 If a curve is obtained by blowing up the intersection of two curves, we call these curves its parents. If a curve is obtained by blowing up a point on one of the curves, this curve is called its parent. The original line at infinity has no parents. Note that other curves may be created afterwards that separate the curve from one or both of its parents.

Definition 2.2 For a given boundary curve $E$ on $Z$, the set of its ancestors $A(E)$ is the smallest set $S$ of the boundary curves that contains its parent(s) and has the property that it contains the parents of every curve in $S$. Note that this set is empty if $E$ is the original line at infinity. Otherwise, it consists of the original line at infinity and all curves on $Z$ that have to be created before $E$.

Note that changing the surface $Z$ above does not change the set of valuations corresponding to the ancestors of $E_{i}$, so we can talk about ancestors of valuations. This notion does depend, however, on the original $\mathbb{P}^{2}$ compactification of $\mathbb{A}^{2}$.

Definition 2.3 A curve $E_{i}$ on $Z$ is called final if there is a sequence of blowups from $X$ to $Z$ such that $E_{i}$ is blown up last. Equivalently, a curve is final if it is not a parent to any curve.

Note that there may be more than one final curve on $Z$, and $\pi_{*}^{-1}(\infty)$ is never final. In what follows, $E_{i}$ is one of the exceptional curves on $Z$. The following easy observations are proven in [3] (cf. also [2]).

\section{Proposition 2.3}

(1) Suppose that when $Z$ was created, $E_{i}$ was created after all of its neighbors in the graph (i.e. all adjacent vertices). Then $E_{i}$ is a final curve.

(2) Suppose $a_{i}=a\left(E_{i}\right) \geq 2$ and it is the largest label among all its neighbors. Then $E_{i}$ is final.

(3) If $a_{i}=1$, then $E_{i}$ is final if and only if it either has only one neighbor, with label 0 , or exactly two neighbors, with labels 1 and 0.

Note that for every divisorial valuation there exists $Z$ such that the center of the valuation is a final curve on $Z$ and all other curves on $Z$ are its ancestors. This can be achieved by refusing to blow up curves that are not ancestors of $E_{i}$ or, equivalently, by blowing up a center of the valuation while it is a point, until it becomes a curve. Note that using this algorithm one can associate to any divisorial valuation and a $\mathbb{P}^{2}$ compactification of $\mathbb{A}^{2}$ a unique surface $Z$.

\section{Determinants of Weighted Forests}

In this section we will construct and use a more sophisticated labeling on the graph of exceptional curves, using the determinants of the matrices related to the Gram matrix of the intersection form. This work is related to the work of Domrina and Orevkov 
(cf. $[4,5])$ and some of our results can be derived from theirs and/or the work of Walter Neumann [10].

In what follows, a weighted tree means a connected graph with no cycles, with weights $a_{i}$ attached to the vertices. A weighted forest is any graph with no cycles, with weights attached to the vertices. To each such graph $\Gamma$ and any ordering of the vertices we will associate a matrix $Q(\Gamma)$ as follows

$$
Q(\Gamma)_{i, j}= \begin{cases}-a_{i}, & \text { if } i=j \\ -1, & \text { if } i \text { th and } j \text { th vertices are connected by an edge } \\ 0, & \text { otherwise. }\end{cases}
$$

Please note that our matrix is essentially minus the standard matrix of a weighted graph. This is because in applications the forest will be obtained from a tree of exceptional curves on $Z$ by removing some vertices and edges, with weights being the self-intersection numbers. So the matrix $Q$ will be related to a Gram matrix of the minus-intersection form. It is somewhat more convenient, because the minusintersection form of the tree of curves on $Z$ is "almost" positive-definite by the Hodge index theorem.

Suppose $\Gamma$ is a weighted forest. Denote by $E(\Gamma)$ and $V(\Gamma)$ the sets of edges and vertices of $\Gamma$, respectively. Denote by $d(\Gamma)$ the determinant of $Q(\Gamma)$ (note that it does not depend on the ordering of the vertices). For every subset $S$ of $E(\Gamma)$ denote by $\operatorname{Sup}(S)$ the set of all vertices that are endpoints of some edge from $S$. A subset of $S$ will be called a matching if no two edges share a vertex (i.e. $|\operatorname{Sup}(S)|=2|S|$ ). Denote by $A(\Gamma)$ the set of all matchings in $\Gamma$. The following lemma provides a useful formula for the determinant $d(\Gamma)$.

Lemma 3.1 In the above notations, if the weight of $v \in V(\Gamma)$ is $a_{v}$,

$$
d(\Gamma)=\sum_{S \in A(\Gamma)}\left((-1)^{|S|} \cdot \prod_{v \in V(\Gamma) \backslash \operatorname{Sup}(S)}\left(-a_{v}\right)\right) .
$$

Proof Using ordering of $V(\Gamma)$, we identify it with $\{1,2, \ldots,|V(\Gamma)|\}$. The determinant $d(\Gamma)$ is the sum of $|V(\Gamma)|$ ! terms corresponding to permutations of $V(\Gamma)$. Note that such term is zero unless the corresponding permutation sends each $i$ to itself or to a vertex $j$ which is connected to $i$ by an edge. Because $\Gamma$ is a forest, the cycle decomposition of such permutation consists of fixed points and transpositions $(i, j)$, where $(i, j)$ are edges of the graph. Obviously, these edges must be disjoint, so the set of such permutations is in one-to one correspondence with $A(\Gamma)$. The formula follows, considering that the sign of such permutation is determined by the parity of the number of transpositions.

We will use the following notation and definitions. Please note that we denote an edge by $(P, Q)$, instead of more standard $\{P, Q\}$ to avoid confusion with a subset $\{P, Q\} \subseteq V(\Gamma)$. 


\section{Definition 3.1}

(1) For $P \in V(\Gamma)$ we denote by $\Gamma_{P}$ the weighted forest obtained from $\Gamma$ by removing $P$ and all edges involving $P$. We define the determinant label $d_{P}$ of the vertex $P$ as $d\left(\Gamma_{P}\right)$.

(2) For $(P, Q) \in E(\Gamma)$ we denote by $\Gamma_{P Q}$ the weighted forest obtained from $\Gamma$ by removing the edge $(P, Q)$. We define the determinant label $d_{P Q}$ of the edge $(P, Q)$ as $d\left(\Gamma_{P Q}\right)$.

(3) For a subset $\{P, \ldots, Q\}$ of $V(\Gamma)$ we denote by $\Gamma_{\{P, \ldots, Q\}}$ the weighted forest obtained from $\Gamma$ by removing all vertices in this subset and all edges involving them. We denote by $d_{P, \ldots, Q}$ the determinant of $\Gamma_{\{P, \ldots, Q\}}$. Note that for an edge $(P, Q)$ the number $d_{P, Q}$ should not be confused with $d_{P Q}$.

The following four properties of the determinants will be used extensively later. We will call them the multiplicativity property, the expansion by vertex formula, the expansion by edge formula, and the weight increment formula.

Lemma 3.2 Suppose $\Gamma$ is a disjoint union of $\Gamma_{1}$ and $\Gamma_{2}$. Then

$$
d(\Gamma)=d\left(\Gamma_{1}\right) \cdot d\left(\Gamma_{2}\right) .
$$

Proof With the appropriate ordering of the vertices, the matrix $Q(\Gamma)$ is blockdiagonal, with blocks being $Q\left(\Gamma_{1}\right)$ and $Q\left(\Gamma_{2}\right)$.

Lemma 3.3 Suppose $P \in V(\Gamma)$. Then

$$
d(\Gamma)=-a_{P} \cdot d_{P}-\sum_{(P, Q) \in E(\Gamma)} d_{P, Q} .
$$

Proof This follows from Lemma 3.1 by breaking up the terms in the sum for $d(\Gamma)$ into classes based on the way the vertex $P$ is involved.

Lemma 3.4 Suppose $(P, Q)$ is an edge of $\Gamma$. Then

$$
d(\Gamma)=d_{P Q}-d_{P, Q}
$$

Proof This follows from Lemma 4.1 by breaking up the terms in the sum for $d(\Gamma)$ into two classes: $(P, Q) \notin S$ and $(P, Q) \in S$.

Lemma 3.5 Suppose $\Gamma^{\prime}$ is obtained from $\Gamma$ by decreasing the weight of $P$ by 1 . Then

$$
d\left(\Gamma^{\prime}\right)=d(\Gamma)+d_{P}
$$


Proof Expanding and contracting by the vertex $P$,

$$
\begin{aligned}
d\left(\Gamma^{\prime}\right) & =\left(-a_{P}+1\right) \cdot d_{P}-\sum_{(P, Q) \in E(\Gamma)} d_{P, Q} \\
& =\left(-a_{P} \cdot d_{P}-\sum_{(P, Q) \in E(\Gamma)} d_{P, Q}\right)+d_{P}=d(\Gamma)+d_{P} .
\end{aligned}
$$

Generalizing the behavior of the weighted graphs of exceptional curves under blowups, we define the blowup operations on the set of all weighted forests as follows. The blowdown is defined as the operation opposite to blowup. This terminology is admittedly unnatural in the abstract graph-theoretic setting. In its original setting of complex algebraic geometry the blowup replaces a point on a surface with the projective line of all directions at that point. The projective line being a Riemann sphere, this resembles blowing up a balloon. This projective line corresponds to the new vertex $R$ in the definition below.

Remark on terminology The unfortunate false reference to explosion/implosion of the blowup/blowdown terminology is likely an oddity of its English version. For instance, the corresponding Russian terms have nothing to do with explosion and implosion, and would be most accurately translated into English as inflation and deflation (or contraction).

\section{Definition 3.2}

(1) Suppose $P$ is a vertex of $\Gamma$. Then the blowup of $\Gamma$ at $P$ is a forest with the set of vertices $V(\Gamma) \cup\{R\}$, the set of edges $E(\Gamma) \cup\{(P R)\} ; a_{R}=-1, a_{P}$ is decreased by 1 , all other weights do not change.

(2) Suppose $(P, Q)$ is an edge of $\Gamma$. Then the blowup of $\Gamma$ at $(P, Q)$ is a forest with the set of vertices $V(\Gamma) \cup\{R\}$, the set of edges $E(\Gamma) \cup\{(P, R),(R, Q)\} \backslash\{(P, Q)\}$; $a_{R}=-1, a_{P}$ and $a_{Q}$ are decreased by 1 , all other weights do not change.

Lemma 3.6 The determinant of a weighted forest is preserved by blowups and blowdowns.

Proof Obviously, it is enough to prove this for the blowups.

Case 1. Suppose $P \in V(\Gamma)$ is blown up, and the new vertex is $R$. Suppose the new graph is $\Gamma^{\prime}$. Expanding by vertex $R$, we get

$$
d\left(\Gamma^{\prime}\right)=d\left(\Gamma_{R}^{\prime}\right)-d\left(\Gamma_{P R}^{\prime}\right)=d\left(\Gamma_{R}^{\prime}\right)-d\left(\Gamma_{P}\right) .
$$

By the weight increment formula,

$$
d\left(\Gamma_{R}^{\prime}\right)=d(\Gamma)+d\left(\Gamma_{P}\right)
$$

which implies the result.

Case 2. Suppose $(P, Q) \in E(\Gamma)$ is blown up, and the new vertex is $R$. Suppose the new graph is $\Gamma^{\prime}$. Because $\Gamma$ is a forest, $\Gamma_{P Q}$ is a disjoint union of two forests. 
We will denote the one that contains $P$ by $P \Gamma$ and the one that contains $Q$ by $Q \Gamma$. Similarly, $\Gamma_{R}^{\prime}$ is a disjoint union of $P \Gamma^{\prime}$ and $Q \Gamma^{\prime}$.

Expanding by the vertex $R$, we get

$$
d\left(\Gamma^{\prime}\right)=d\left(\Gamma_{R}^{\prime}\right)-d\left(\Gamma_{P, R}^{\prime}\right)-d\left(\Gamma_{Q, R}^{\prime}\right) .
$$

By the multiplicativity property,

$$
\begin{aligned}
d\left(\Gamma_{R}^{\prime}\right) & =d\left(P \Gamma^{\prime}\right) \cdot d\left(Q \Gamma^{\prime}\right), \\
d\left(\Gamma_{P, R}^{\prime}\right) & =d\left(P \Gamma_{P}^{\prime}\right) \cdot d\left(Q \Gamma^{\prime}\right), \\
d\left(\Gamma_{Q, R}^{\prime}\right) & =d\left(Q \Gamma_{Q}^{\prime}\right) \cdot d\left(P \Gamma^{\prime}\right) .
\end{aligned}
$$

Note that $P \Gamma_{P}^{\prime}=P \Gamma_{P}$. By the weight increment formula,

$$
d\left(P \Gamma^{\prime}\right)=d(P \Gamma)+d\left(P \Gamma_{P}\right) .
$$

Likewise, $d\left(Q \Gamma^{\prime}\right)=d(Q \Gamma)+d\left(Q \Gamma_{Q}\right)$. Putting this together, we get

$$
\begin{aligned}
d\left(\Gamma^{\prime}\right)= & \left(d(P \Gamma)+d\left(P \Gamma_{P}\right)\right)\left(d(Q \Gamma)+d\left(Q \Gamma_{Q}\right)\right)-d\left(P \Gamma_{P}\right)\left(d(Q \Gamma)+d\left(Q \Gamma_{Q}\right)\right) \\
& -d\left(Q \Gamma_{Q}\right)\left(d(P \Gamma)+d\left(P \Gamma_{P}\right)\right) \\
= & d(P \Gamma) d(Q \Gamma)-d\left(P \Gamma_{P}\right) d\left(Q \Gamma_{Q}\right) .
\end{aligned}
$$

On the other hand, expanding $d(\Gamma)$ by the edge $(P, Q)$, we get

$$
d(\Gamma)=d\left(\Gamma_{P Q}\right)-d\left(\Gamma_{P, Q}\right) .
$$

By the multiplicativity property, this equals the above expression for $d\left(\Gamma^{\prime}\right)$, which completes the proof of the lemma.

Corollary 3.1 The determinants of vertices and edges are preserved by blowups and blowdowns that do not destroy them.

Proof Most blowups and blowdowns that do not destroy the corresponding vertex or edge do not involve it. For those that do, we note that blowing up an edge $(P, Q)$ and then removing $P$ is the same as removing $P$ and then blowing up $Q$. Also, when a vertex $P$ is blown up and then removed, the determinant of the resulting graph is the same as the determinant of the original graph with vertex $P$ removed, because the new vertex has weight -1 .

The above corollary means that just like the coefficients of the $\bar{K}$ labels, the determinant labels on vertices and edges of the graph of exceptional curves on $Z$ do not change once the point or edge is created (the edge, however, may get destroyed). While we are ultimately more interested in the labels of vertices, we need to keep track of the edges as well. The following lemma is very significant, as it describes the determinants of vertices and edges formed after the blowup in terms of the nearby determinants and the determinant of the entire graph. The existence of these formulas is not intuitively obvious, and the formulas themselves are very important for applications. 
Lemma 3.7 Suppose $\Gamma^{\prime}$ is obtained from $\Gamma$ by a single blowup. Suppose $d\left(\Gamma^{\prime}\right)=$ $d(\Gamma)=d$.

Case 1. Suppose $P \in V(\Gamma)$ is blown up, and the new vertex is $R$. Then $d_{R}=d_{P}+d$ and $d_{P R}=d_{P}+d$. Here $d_{R}$ and $d_{P R}$ are the determinants of a vertex and an edge in $\Gamma^{\prime}$, and $d_{P}$ is the determinant in $\Gamma$ or $\Gamma^{\prime}$ (which are the same by the previous lemma).

Case 2. Suppose $(P, Q) \in E(\Gamma)$ is blown up, and the new vertex is $R$. Then

$$
\begin{aligned}
d_{R} & =2 d_{P Q}+d_{P}+d_{Q}-d, \\
d_{P R} & =d_{P}+d_{P Q}, \\
d_{Q R} & =d_{Q}+d_{P Q} .
\end{aligned}
$$

Proof In Case 1, one immediately sees that $d_{P R}=d_{R}$, because the weight of $R$ is $(-1)$. Then the weight increment formula implies that $d_{R}=d+d_{P}$.

In Case 2, using the notation and work from the previous lemma, we have

$$
\begin{aligned}
d_{R} & =d\left(\Gamma_{R}^{\prime}\right)=d\left(P \Gamma^{\prime}\right) d\left(Q \Gamma^{\prime}\right)=\left(d(P \Gamma)+d\left(P \Gamma_{P}\right)\right) \cdot\left(d(Q \Gamma)+d\left(Q \Gamma_{Q}\right)\right) \\
& =d_{P Q}+d_{P}+d_{Q}+d\left(P \Gamma_{P}\right) d\left(Q \Gamma_{Q}\right)=d_{P Q}+d_{P}+d_{Q}+\left(d_{P Q}-d\right) .
\end{aligned}
$$

This proves the formula for the vertex. For the edges, it is enough to prove the formula for $d_{P R}$.

$$
d_{P R}=d\left(P \Gamma^{\prime}\right) \cdot d(Q \Gamma)=\left(d(P \Gamma)+d\left(P \Gamma_{P}\right)\right) d(Q \Gamma)=d_{P Q}+d_{P} .
$$

\section{Two labelings together}

The original motivation for the $\bar{K}$ and the determinant labeling comes from looking for possible counterexamples to the Jacobian conjecture (cf. [2]). We are especially interested in the curves for which both the $\bar{K}$ label and the determinant label are negative. For example, all curves that are mapped to the line at infinity on $\mathbb{P}^{2}$ (called curves of type 1 in [3]), are of this kind, as a consequence of the Ample Ramification Theorem of [3].

We begin with a new definition.

Definition 4.1 For a curve $P$ (or a corresponding valuation) we will call the sum of its $\bar{K}$ label and its determinant label its content, to be denoted $c_{P}$.

\section{Lemma 4.1}

(1) In the notation of Lemma 3.7, if a point on one exceptional curve is blown up, the content of the new curve equals the content of its parent. If the point of intersection of curves $P$ and $Q$ is blown up into the curve $R$, then

$$
c_{R}=c_{P}+c_{Q}+\left(2 d_{P Q}+1\right) .
$$

(2) The content $c_{P}$ is always odd. 
Proof The first statement is a consequence of Lemma 3.7 and Proposition 2.1. The second statement then follows by induction.

The following result is interesting in its own right, and its proof also illustrates a method that will be used in subsequent theorems.

Theorem 4.1 Every $\bar{K}$-negative curve $P$ with negative $d_{P}$ has a $\bar{K}-0$ curve as its ancestor.

Proof For a curve $P$, we will denote its $\bar{K}$ label by $b_{P}$. We will identify the curves with vertices of the graphs. Suppose we perform any number of blowups without creating a $\bar{K}-0$ curve. This means that $b_{P} \leq-1$ for all curves $P$ involved. We will prove by induction on the number of blowups the following two conditions.

(1) For all vertices $P, c_{P} \geq-1$. Note that this implies that $d_{P} \geq 0$.

(2) For all edges $P Q, d_{P Q} \geq 0$.

The base of the induction is easy to check. To prove the step, we need to consider two cases of blowups. Note that $d(\Gamma)=d=-1$.

Case 1 . Blowing up a vertex $P$ to get a new vertex $R$.

$$
c_{R}=c_{P} \geq-1 ; \quad d_{P R}=d_{R} \geq 0 .
$$

Case 2. Blowing up an edge $P Q$ to get a new vertex $R$.

$$
c_{R}=c_{P}+c_{Q}+\left(2 d_{P Q}+1\right) \geq(-1)+(-1)+(0+1)=-1 .
$$

For the edges,

$$
d_{P R}=d_{P}+d_{P Q} \geq 0+0=0 .
$$

Example 4.1 Starting from $X=\mathbb{P}^{2}$, we blow up a point at infinity, and then a point on the new curve. The resulting surface has the following graph of exceptional curves.

$\begin{array}{rrrrr}1 & 0 & 0 & -1 & -1 \\ 0 & 0 & & 0 \\ -2 & & -1 & & 0 \\ 0 & & -2 & & -1\end{array}$

Here the labels above the graph are the edge and vertex determinants, the labels right below the graph are the $\bar{K}$-labels, and the labels on the bottom line are the selfintersection labels. If one blows up the point of intersection of the last two blown-up curves, one gets a curve with negative $\bar{K}$-label and negative determinant label. Other such curves could then be obtained by further blowups.

The following theorem shows that, up to a polynomial automorphism of the original $\mathbb{A}^{2}$, every curve with negative $\bar{K}$ and determinant labels has an ancestor as in the example above. 
Theorem 4.2 Suppose $P \subset Z \backslash \mathbb{A}^{2}$. Suppose $E$ is the first $\bar{K}-0$ curve that has to be created to create $P$ (the oldest $\bar{K}-0$ ancestor of $P$ ). Then there exists a sequence of blowups and blowdowns at infinity that transform $Z$ into $Z^{\prime}$, which has the same graph as the example above, with $E$ being the $\bar{K}-0$ curve. (Note that the pullback of the line at infinity on $Z^{\prime}$ may differ from the original pullback of infinity on $Z$ ).

Proof We will follow the way $P$ was created, changing the pullback of infinity as needed, to decrease the number of ancestors of $E$. The key idea is that one can rule out the possibility of creating $P$ in some choices of blowups.

We start with blowing up a point at infinity. If then the new point is blown-up on the last curve, we are in the situation of the example above, and the result is obviously true. So the only non-trivial option left is to blow up the point of intersection of the two curves, creating the following graph:

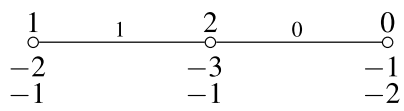

The next step in the sequence of creation of $E$ must be either blowing up the left edge, the middle point, or the right edge of the above graph.

If the left edge is blown up, we end up in the part of the graph with all positive edge labels, and the inequality $c_{Q} \geq 0$ is satisfied for all the vertices (except the one that used to be the middle vertex). The induction argument, similar to the one in the proof of the last theorem, implies that even if we create a $\bar{K}-0$ curve, its determinant label, and the label of the edge created with it, will be non-negative. Since we have to go back to the "negative $\bar{K}$ " territory, we will never get a curve with negative $\bar{K}$ and determinant labels.

If the middle point is blown up (which corresponds to blowing up a new point on the last blown-up curve) then the newly blown-up curve can serve as a new pullback of infinity, which is closer to $E$ (i.e. $E$ can be created from it by fewer blowups).

So the only case left is when the right edge is blown up. After that we again have three possibilities. Blowing up the left edge can be discarded the same way as above. If we blow up the last vertex, we will be forced to blow up the newly blown-up vertex, creating a new curve that can serve as a pullback of infinity.

In general, suppose the creation sequence for $E$ starts with blowing up the right edge exactly $k$ times. This leads to the following graph:

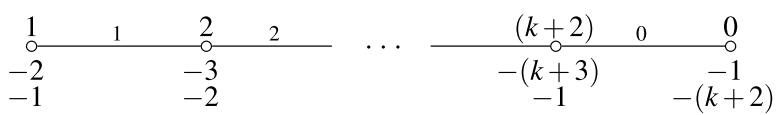

After this, we are forced to blow up the second from the right vertex, and then blow up the resulting vertex, and so on, until we get a curve with the $\bar{K}$-label ( -2$)$. Notice that this curve $Q$ can serve as a new pullback of infinity, because all other curves can be contracted one after another to a smooth surface, which has to be $P^{2}$, since the anticanonical class is $-3 Q$ and $Q^{2}=1$. Note that this $Q$ is closer to $E$ that the original pullback of infinity. 
We are now going to prove our main theorem. It would be nice to have a conceptual proof, but at this time we have to resort to a case-by-case analysis of the combinatorics of weighted graphs.

Theorem 4.3 (Main theorem) Suppose $a, b \in \mathbb{Z}$ are arbitrary fixed numbers. Then there exists a number $N \in \mathbb{N}$ such that every divisorial valuation at infinity with $\bar{K}$ label $a$ and determinant label $b$ is given by a curve on a compactification of $\mathbb{A}^{2}$ that can be obtained from a $\mathbb{P}^{2}$ by no more than $N$ blowups.

Proof of main theorem Suppose $v$ is a divisorial valuation at infinity. We compactify $\mathbb{A}^{2}$ to $X=\mathbb{P}^{2}$ and consider the center of $v$ on $X$. Unless it is the line at infinity, it is a point on the line at infinity. We blow it up, and keep blowing it up until it becomes a curve. Since we are considering the valuations modulo automorphisms of $\mathbb{A}^{2}$, we will choose such $\mathbb{P}^{2}$ compactification of $\mathbb{A}^{2}$ that the valuation $v$ has the fewest possible number of ancestors. We will only keep track of the $\bar{K}$ labels and the determinant labels, generally indicated below and above, respectively; the curve blown up last is indicated by " $\otimes$ " instead of "०". After the first blowup, the graph looks as follows.

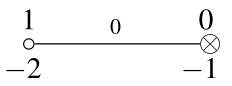

Depending on the next blowup, we have two cases.

Case 1.

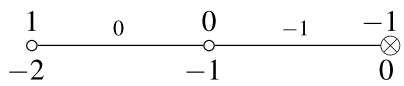

Case 2.

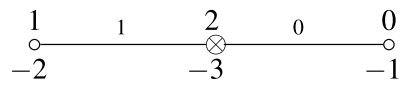

Lemma 4.2 In Case 1, the following is true for all subsequent blowups.

(1) All future determinants of vertices are negative.

(2) All future determinants of edges are negative.

Proof of lemma The proof is by induction; the base is obvious, and the step follows from Lemma 3.7.

As a corollary of the above and Lemma 3.7, the determinant of the last vertex strictly decreases at every step. Thus the number of steps in this case is bounded by $|b|$.

Case 2 is somewhat more complicated. It can be subdivided into three sub-cases.

Case $2 \mathrm{a}$. A point is blown up on the last curve. In this case the new curve can be taken as the new line at infinity, contracting all the other curves. This corresponds to an automorphism of $\mathbb{A}^{2}$; because we chose the $\mathbb{P}^{2}$ compactification with the smallest number of ancestors of $v$, this case is impossible. 
Case 2b. The left edge is blown up:

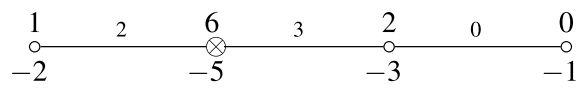

This will be treated below as part of the more general case, for $n=2$. Case $3 c$. The right edge is blown up:

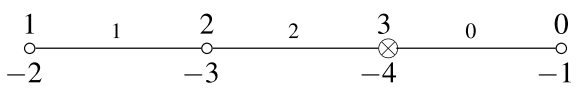

Here we can again either blow up a new point on the last curve, blow up the edge to the left of the last curve or the edge to the right. In the first case there is a compactification of $\mathbb{A}^{2}$ such that $v$ has smaller number of ancestors (see the end of the proof of Theorem 4.2). This contradicts our assumption on minimality of the number of ancestors of $v$. In the second case, we get the following graph:

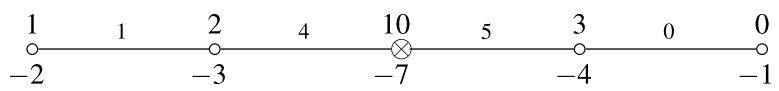

In the last case, we again have three possibilities ... ultimately, after a finite number of blowups of the right edge we will either have found the curve for $v$ or we will have to blow up a point on the last curve, or the left edge. The first case is again impossible by the minimality of the number of ancestors of $v$, and the last case gives the following graph:

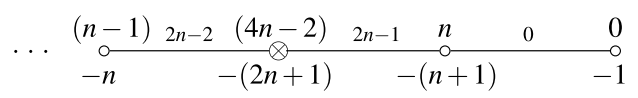

Incorporating the previously considered cases, we can have here any $n \geq 2$. Note that if we denote the last curve by $P$, then

$$
c_{P}=(4 n-2)-(2 n+1)=2 n-3 \geq 1 .
$$

In the subsequent blowups, the $\bar{K}$ labels of the blown-up curves are non-positive at first. If the $\bar{K}$ label of $v$ is positive, then at some point these labels become positive. By Proposition 2.1, once the $\bar{K}$ labels become positive, they stay positive. The following lemma describes what happens while the $\bar{K}$ labels are negative. The increase or decrease of the labels refers to the sequences of the labels of the curves blown up during the steps of the process.

Lemma 4.3 In the notations above, we have the following for the subsequent blowups, while the $\bar{K}$ labels are non-positive:

(1) All determinant labels of the vertices and edges are positive, except for the rightmost edge and vertex on the above graph.

(2) All $c_{P}$ created during these blowups are at least 1.

(3) The sequence of $c_{P}$ 's never decreases; at every step when it stays the same, the $\bar{K}$ label increases. 
Proof of lemma The proof is by induction, and the base is obvious. Suppose all conditions are satisfied after $k$ blowups. Consider the $(k+1)$-st blown-up curve, $R$.

If $R$ has one parent, then the condition 3 follows from Lemma 4.1 and Proposition 2.1. The condition 2 follows from Lemma 4.1 and the induction hypothesis. The condition 1 for $R$ follows from $c_{R}$ being at least 1 and the $\bar{K}$ label of $R$ being non-positive. Then the condition 1 for the newly formed edge follows from the determinant label of the edge being equal to the label of $R$ (see Lemma 3.7).

If $R$ has two parents, $P$ and $Q$, then by Lemma 4.1 and positivity of $d_{P Q}, d_{P}$, and $d_{Q}$,

$$
c_{R}=c_{P}+c_{Q}+\left(2 d_{P Q}+1\right) \geq \max \left(c_{P}, c_{Q}\right)+4,
$$

thus the content of $R$ is strictly greater than that of the previous curve. By induction, this implies condition 2 . The condition 1 follows from the induction hypothesis and Lemma 3.7.

If $a \leq 0$, the condition 3 implies that $2 n+3 \leq(a+b)$, thus for given $a$ and $b$ we have finitely many possible $n$. For each of these $n$, we can perform only a finite number of blowups without increasing $c_{P}$, since we have to keep the $\bar{K}$ label nonpositive. So after a bounded number of steps we have to increase $c_{P}$. After that we again have only a finite number of possible graphs, so the number of blowups until $c_{P}$ has to increase twice is also bounded, and so on. Note that the total number of times $c_{P}$ can increase is bounded as well, by $(a+b-1) / 2$, thus we have a finite number of possible graphs for given $a$ and $b$, for $a \leq 0$.

If $a \geq 1$, then at some step of the blowup process we must create for the first time a vertex with positive $\bar{K}$ label. Call this vertex $Q$. Note that its $\bar{K}$ label must be 1 , and it has one parent, with $\bar{K}$ label 0 . By the above lemma, the content of its parent, which is also its content, is at least $2 n-3$. Therefore, $d_{Q} \geq 2 n-4 \geq 0$. We then blow up $m \geq 0$ times the edge connecting the vertices with the $\bar{K}$ labels 0 and 1 before creating (if $a \geq 2$ ) a vertex with the $\bar{K}$ label 2 . The following lemma describes this process.

Lemma 4.4 In the notation above, after $m$ blowups the graph looks as follows, if $d_{Q}=c$ :

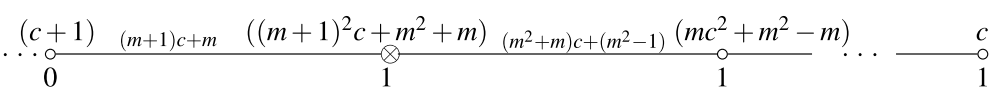

Proof of lemma The proof is just a calculation, using induction on $m$ and Lemma 3.7. Please note that when $m=0$ the above picture degenerates to just two left vertices.

After this the vertex $R$ with $\bar{K}$ label 2 can be created in two different ways.

If $R$ has one parent, then at the step when $R$ was created the graph looked as below, with $d_{R}=(m+1)^{2} c+m^{2}+m-1$ :

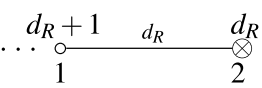


If $R$ has two parents, then the graph looked like this:

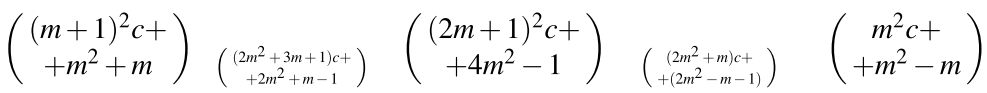

$$
\begin{aligned}
& 12+2-1
\end{aligned}
$$

Note that this can only happen if $m \geq 1$.

Once $R$ is created, the sequence of $\overline{\bar{K}}$ labels will be strictly increasing, so we will have to get to the curve of $v$ in at most $(a-2)$ steps. This gives for every $a$ a finite number of possibilities for the sequence of blowups from $R$ to the vertex of $v$. Each such sequence corresponds to a tree, with vertices and edges labeled by the respective determinants, calculated from Lemma 3.7.

Lemma 4.5 In both of the situations described above, the following are true both for the determinants of vertices and the determinants of edges for all subsequent blowups.

(1) Each of these determinants is an integer polynomial in $c=d_{Q}$ and $m$, linear in $c$ and quadratic in $m$.

(2) For any fixed $c \geq 0$ each of these polynomials is a strictly increasing functions of $m$, for $m \geq 0$ in the one-parent situation and $m \geq 1$ in the two-parent situation.

(3) For any fixed $m \geq 0$ in the one-parent situation and $m \geq 1$ in the two-parent situation, each of these polynomials is a strictly increasing function of $c$, for $c \geq 0$.

Proof of lemma This follows from Lemma 3.7 by induction on the number of blowups after creation of $R$. Note that the operations on polynomials described in Lemma 3.7 preserve properties 2 and 3.

Thus, since $b$ is fixed, for each of the finite number of possible blowup sequences after $R$ we have only finitely many choices for $d_{Q}$ and $m$. The determinant label of the last curve with the $\bar{K}$ label 0 (the parent of $Q$ ) is $d_{Q}+1$, so it is bounded. Therefore $n$ is bounded and from the above discussion of the $a \leq 0$ case the number of steps to create $Q$ is bounded, which completes the proof of the theorem.

Note that the above proof does not provide an effective estimate $N(a, b)$. However, for some fixed small $a$ and $b$ one can find the moduli space of valuations with labels $a$ and $b$ explicitly. As an example, we get the following nice characterization of the line at infinity.

Theorem 4.4 Suppose $v$ is a divisorial valuation at infinity, such that its $\bar{K}$ label is -2 and its determinant label is 1 . Then, up to an automorphism of $\mathbb{A}^{2}$, its center is the line at infinity.

Proof As in the above theorem, consider a $\mathbb{P}^{2}$ compactification of $\mathbb{A}^{2}$ so that $v$ has the smallest number of ancestors. Suppose this number is positive, that is, we have 
to perform blowups to get the curve of $v$. Then Case 1 is impossible because all the curves there have negative determinant. Case 2 is impossible because for the valuations with negative $\bar{K}$ labels the content must be at least 1 .

\section{Several $\bar{K}$-negative curves}

We are now interested in several $\bar{K}$-negative curves, with the condition that removing them all from the graph of exceptional curves produces a graph with negative determinant. This is motivated by the curves of type 1 of [3] (cf. also [2]).

First, we need to introduce some new notation. Suppose $X=\mathbb{P}^{2}$ is the standard compactification of $\mathbb{A}^{2}, Z$ is obtained from $X$ by a sequence of blowups outside $\mathbb{A}^{2}$; $\Gamma$ is the (dual) graph of the exceptional curves on $Z, E=\pi_{*}^{-1}(\infty)$, where $\pi: Z \rightarrow X$ is the natural map. Suppose $P$ is an exceptional curve on $Z$, and a vertex of $\Gamma$. We denote by $d_{P}^{\prime}$ the determinant of $\Gamma_{E, P}$. We denote by $u_{P}$ the coefficient of $P$ in the formula that expresses $\pi^{*}(\infty)$ in terms of the exceptional curves on $Z: \pi^{*}(\infty)=$ $E+\sum_{P \neq E} u_{P} P$. Note that $u_{P}$ is a natural number.

Lemma 5.1 In the above notation, $u_{P}^{2}=d_{P}+d_{P}^{\prime}$.

Proof While an inductive argument is possible, the following direct proof is easier. Define $L=E+\cdots+u_{P} P+\cdots$, the class of the pullback of $(\infty)$ on $Z$. Note that $L$ intersects by 1 with $E$ and by 0 with all other exceptional curves. If we change a basis of the lattice of the divisor classes on $Z$, replacing $P$ by $L$, the Gram matrix of the minus-intersection form will be multiplied by $u_{P}^{2}$. Thus

$$
(-1) \cdot u_{P}^{2}=\operatorname{det}\left(\begin{array}{ccc}
\left(-E^{2}\right) & \ldots & -1 \\
\ldots & & \\
-1 & & -1
\end{array}\right) \text {, }
$$

where the last row and column of the matrix on the right correspond to $L$ and the first correspond to $E$. Note that all the coefficients in the last row and column are zero unless indicated, and that removing these row and column produces the matrix of $\Gamma_{P}$. Using cofactor expansion w.r.t. the last row, and then (for one of the terms) the first row, we get $-u_{P}^{2}=-d_{P}-d_{P}^{\prime}$.

Lemma 5.2 Suppose $P$ and $Q$ are on the different sides of $E$ (i.e. E belongs to the pass in the tree $\Gamma$ that connects $P$ and $Q$ ). Then the determinant $d_{P, Q}$ of the graph $\Gamma_{P, Q}$ is given by the following formula:

$$
d_{P, Q}=u_{P} u_{Q}-d_{P} d_{Q} .
$$

Proof We will denote $d_{P, Q}$ by ${ }_{P} d_{Q}$. The notation $C_{1} C_{2} d_{Q}$ will stand for the determinant of the graph obtained by removing the vertex $Q$ ad the edge $C_{1} C_{2}$.

We first perform a sequence of blowups to create $Q$, without producing any ancestors of $P$. Then we perform a sequence of blowups that creates $P$, keeping track of the determinants of vertices and edges of the new graph with the point $Q$ removed. Note the following: 
(1) ${ }_{E} d_{Q}=d_{Q}^{\prime}$.

(2) $d\left(\Gamma_{Q}\right)=d_{Q}$

(3) For all ancestors $C$ of $P$ and all edges $C_{1} C_{2}$ involved in creating $P$, their determinant labels ${ }_{C} d_{Q}$ and $C_{1} C_{2} d_{Q}$ are linear combinations of $d_{Q}^{\prime}$ and $d_{Q}$. Let us denote the coefficients of these linear combinations by $a_{C}, b_{C}, a_{C_{1} C_{2}}, b_{C_{1} C_{2}}$ so that

$$
{ }_{C} d_{Q}=a_{C} \cdot d_{Q}^{\prime}+b_{C} \cdot d_{Q}, \quad C_{1} C_{2} d_{Q}=a_{C_{1} C_{2}} \cdot d_{Q}^{\prime}+b_{C_{1} C_{2}} \cdot d_{Q} .
$$

(4) From the formulas for the determinants of blowups (Lemma 3.7) the coefficients $a_{C}$ and $a_{C_{1} C_{2}}$ behave like $d_{C}$ and $d_{C_{1} C_{2}}$ for a graph with the determinant $d=0$, and initial value 1 . By induction, one can easily prove that $a_{C}=d_{C}+d_{C}^{\prime}$, which equals $u_{C}^{2}$.

(5) From the formulas for the determinants of blowups (Lemma 3.7) the coefficients $b_{C}$ and $b_{C_{1} C_{2}}$ behave like $d_{C}$ and $d_{C_{1} C_{2}}$ for a graph with the determinant $d=1$, and initial value 0 . By induction, one can easily prove that $b_{C}=d_{C}^{\prime}$.

(6) So, ${ }_{C} d_{Q}=u_{C}^{2} \cdot d_{Q}^{\prime}+d_{Q}^{\prime}=u_{C}^{2}\left(u_{Q}^{2}-d_{Q}\right)+\left(u_{C}^{2}-d_{C}\right) d_{Q}=u_{C}^{2} u_{Q}^{2}-d_{C} d_{Q}$.

Lemma 5.3 In the above notation, for a curve $P$ with $\bar{K}$ label $\bar{k}_{P}<0$ and $d_{P}<0$ the following inequality is satisfied:

$$
u_{P}^{2} \geq\left|d_{P}\right|
$$

Proof Because $d_{P}<0$, we need to prove that $u_{P}^{2}+d_{P} \geq 0$. Note that $u_{P}^{2}+d_{P}=$ $2 d_{P}+d_{P}^{\prime}$. For all ancestors $C$ of $P$, define $l_{C}=2 d_{C}+d_{C}^{\prime}$ and $l_{C_{1} C_{2}}=2 d_{C_{1} C_{2}}+$ $d_{C_{1} C_{2}}^{\prime}$. The labels $l_{C}$ and $l_{C_{1} C_{2}}$ behave like those in the graph with $d=-1$. Like in the proof of Theorem 4.1, one can prove by induction the following two statements:

(1) $l_{C}+\bar{k}_{C} \geq-1$.

(2) $l_{C_{1} C_{2}} \geq 0$.

So $l_{P}+\bar{k}_{P} \geq-1$. Because $\bar{k}_{P}<0, l_{P} \geq 0$.

Theorem 5.1 Suppose $P$ and $Q$ have negative $\bar{k}$ labels and determinant labels and lie on the different sides of $E$. Then $d_{P, Q} \geq 0$.

Proof This follows directly from Lemmas 5.2 and 5.3.

Corollary 5.1 Suppose $\left\{E_{1}, \ldots, E_{k}\right\}$ is any collection of exceptional curves of $Z$ with negative $\bar{k}$ labels, such that removing them all from the graph of exceptional curves produces a graph with negative determinant. Then they all lie on the one side of $E$.

Remark 5.1 Corollary 5.1 can be applied to the set of all curves on any counterexample $Z$ to the two-dimensional Jacobian conjecture that are mapped to the (same) line at infinity on $\mathbb{P}^{2}$ (curves of typ 1 as defined in [3]). As a result, we conclude that all these curves are on one side of $\pi_{*}^{-1}(\infty)$. 
Acknowledgements The research of the author was supported in part by the NSA grants H98230-08-10129, H98230-06-1-0034 and H98230-11-1-0148.

This paper is dedicated to the memory of V.A. Iskovskikh, who introduced the author to the beauty of birational geometry. The author wishes to thank his colleagues at the University of Pittsburgh for their interest and support. The author also wishes to thank anonymous referees for several helpful comments.

\section{References}

1. Abhyankar, S.S.: Lectures on Expansion Techniques in Algebraic Geometry. Notes by Balwant Singh. Tata Institute of Fundamental Research Lectures on Mathematics and Physics, vol. 57. Tata Institute of Fundamental Research, Bombay (1977), iv+168 pp.

2. Borisov, A.: On resolution of compactifications of unramified planar self-maps. Preprint, Web address: http://front.math.ucdavis.edu/1110.5118

3. Borisov, A.: On the Stein factorization of resolutions of Jacobian self-maps of $\mathbb{P}^{2}$

4. Domrina, A.V., Orevkov, S.Y.: Four-sheeted polynomial mappings of $C^{2}$. I. The case of an irreducible ramification curve. Mat. Zametki 64(6), 847-862 (1998) (Russian). Translation in Math. Notes 64(56), 732-744 (1999)

5. Domrina, A.V.: Four-sheeted polynomial mappings of $C^{2}$. II. The general case. Izv. Ross. Akad. Nauk Ser. Mat. 64(1), 3-36 (2000) (Russian). Translation in Izv. Math. 64(1), 1-33 (2000)

6. Favre, C., Jonsson, M.: The Valuative Tree. Lecture Notes in Mathematics, vol. 1853, Springer, Berlin (2004), xiv+234 pp.

7. Favre, C., Jonsson, M.: Eigenvaluations. Ann. Sci. Éc. Norm. Super. 40, 309-349 (2007)

8. Favre, C., Jonsson, M.: Dynamical compactifications of $\mathbb{C}^{2}$. Ann. Math. (2) 173(1), 211-248 (2011)

9. Mondal, P.: Compactifications of $\mathbb{C}^{2}$ via pencils of jets of curves. C.R. Math. Acad. Sci. Soc. R. Can. 34(3), 79-96 (2012)

10. Neumann, W.D.: On bilinear forms represented by trees. Bull. Aust. Math. Soc. 40, 303-321 (1989)

11. Oscar, Z.: The compactness of the Riemann manifold of an abstract field of algebraic functions. Bull. Am. Math. Soc. 50, 683-691 (1944) 